\section{Peroral endoscopic myotomy (POEM) for diffuse esophageal spasm}

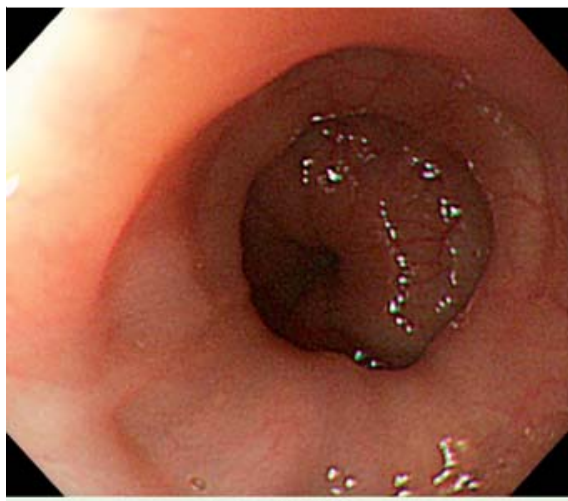

Before POEM

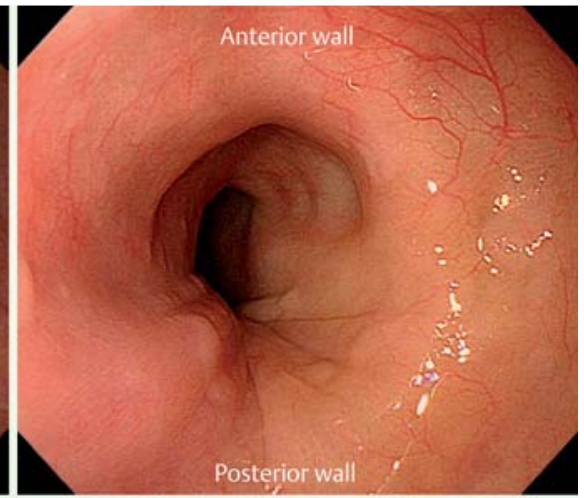

After POEM
Fig. 1 Left image shows the abnormal simultaneous contractions before peroral endoscopic myotomy. The scope passed through the contraction segment with moderate resistance. Post-procedural esophagoscopy showed that the contractions were not seen on the anterior side of the muscle incision (right image).

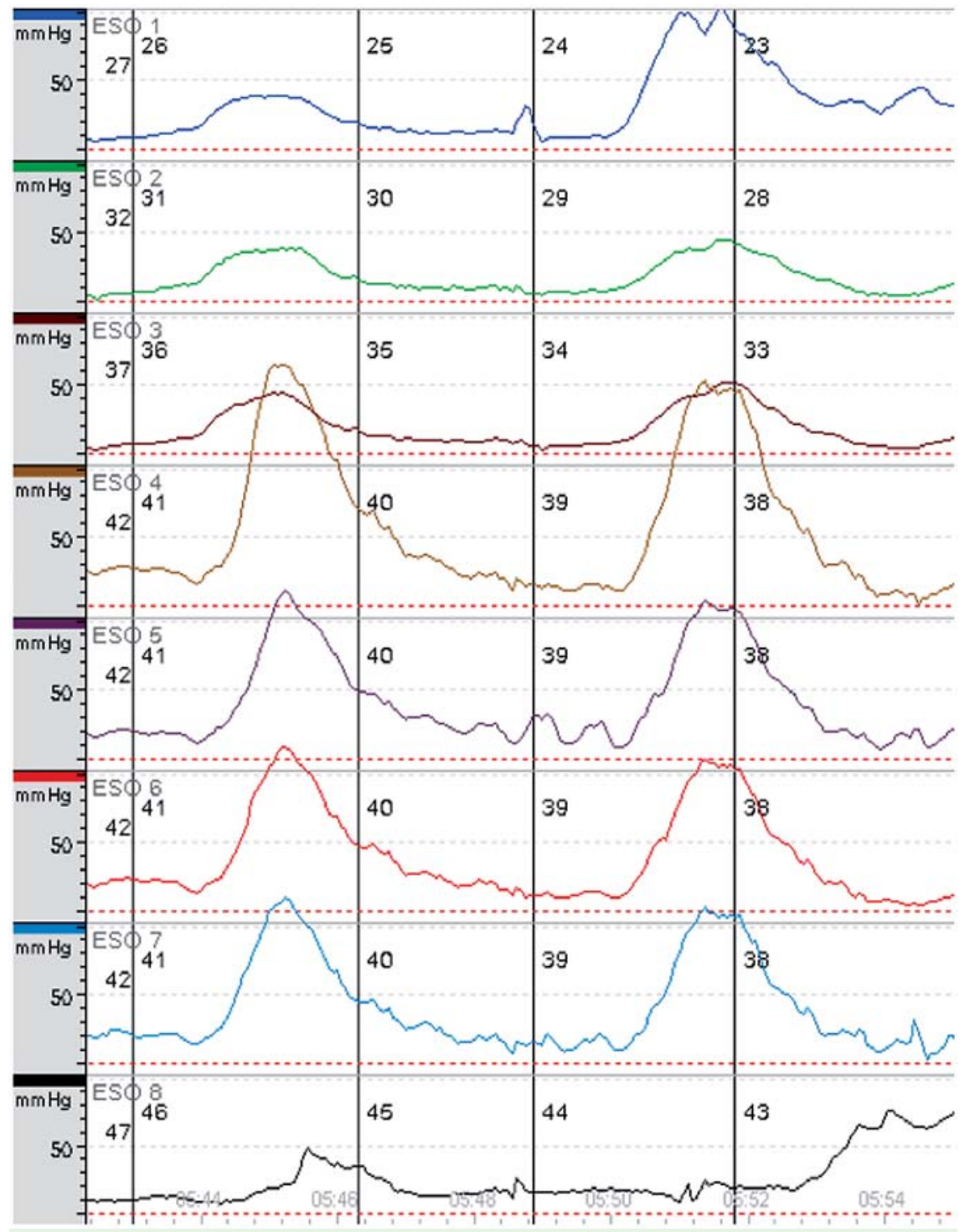

Fig. 2 Esophageal manometry showed simultaneous contractions, with a high pressure of $119.0 \mathrm{mmHg}$ on average.
Diffuse esophageal spasm (DES) is a rare esophageal dysmotility condition characterized by simultaneous contractions of the distal esophagus and manifested by dysphagia and chest pain. Pharmacological therapy, endoscopic interventions, and surgical myotomy have been linked to various outcomes $[1,2]$. Recently, peroral endoscopic myotomy (POEM) has been introduced as an effective and less invasive treatment for achalasia [3]. We report here our clinical experiences of POEM for DES.

Patient 1 was an 84-year-old woman with a 5-year history of dysphagia accompanied by excessive weight loss. Esophagogastroduodenoscopy (EGD) and manometry revealed severe simultaneous contractions in the lower esophagus ( $\bullet$ Fig. 1 and $\bullet$ Fig. 2) and POEM was applied. A myotomy of $15.0 \mathrm{~cm}$ in length was performed longitudinally to include each contraction segment ( Fig. 3). Subjective dysphagia symptom scores and pressure study were markedly improved ( $\bullet$ Fig. 2 and Fig.4). EGD after POEM showed complete absence of abnormal contractions in the incised esophagus ( $\bullet$ Fig. 1 ). Patient 2 was a 79-year-old man with a 20year history of dysphagia, vomiting, and excessive weight loss. Endoscopic passage was impossible during the simultaneous contractions. Esophagography showed corkscrew-shaped contractions and blockage of barium passage at the proximal portion of contractions ( Fig.5). The maximum pressure at the contraction site was $256.0 \mathrm{mmHg}$. A $10-\mathrm{cm}$ myotomy was successfully performed according to the findings of the Esophagography and manometry. Esophagography after POEM revealed complete absence of abnormal contractions on the incised anterior esophagus ( $\bullet$ Fig.5).

Details of the results are shown in - Table 1. No recurrence of dysphagia or postoperative gastroesophageal reflux disease has been observed in either patient since the procedures (5 and 6 months, respectively, at the time of writing).

The length of myotomy was decided according to the findings of esophagography and manometry. As expected, the patients' symptoms disappeared completely. In contrast to surgical myotomy, which requires additional antireflux procedures, POEM does not cause any destruction of the tissues surrounding the esophagogastric junction. These topics should be investigated further. 


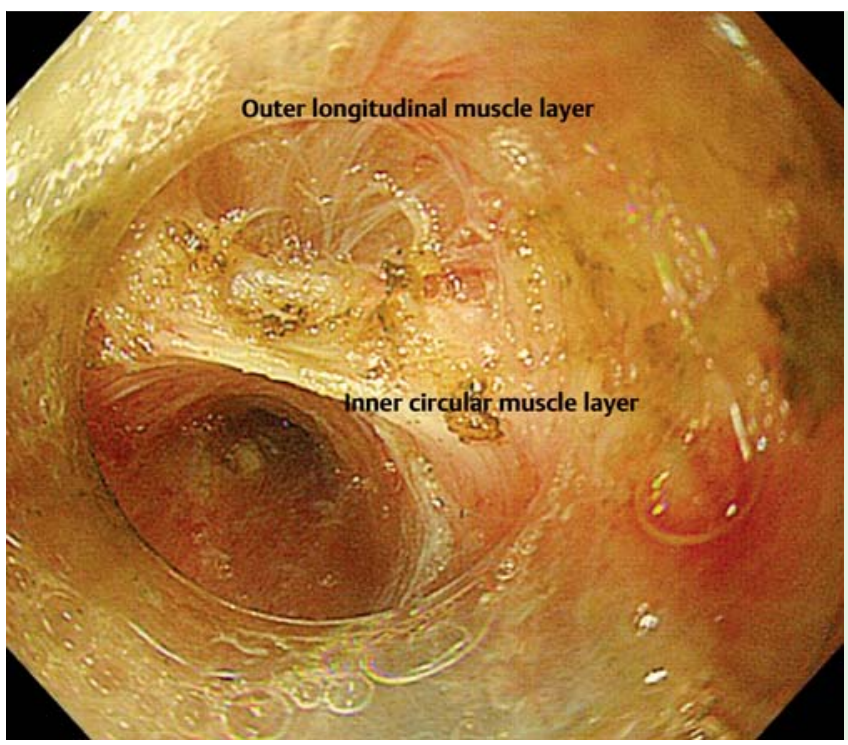

Fig. 3 Peroral endoscopic myotomy was successfully performed. After creation of mucosal entry, the inner circular muscle was incised along a $10-\mathrm{cm}$ length.

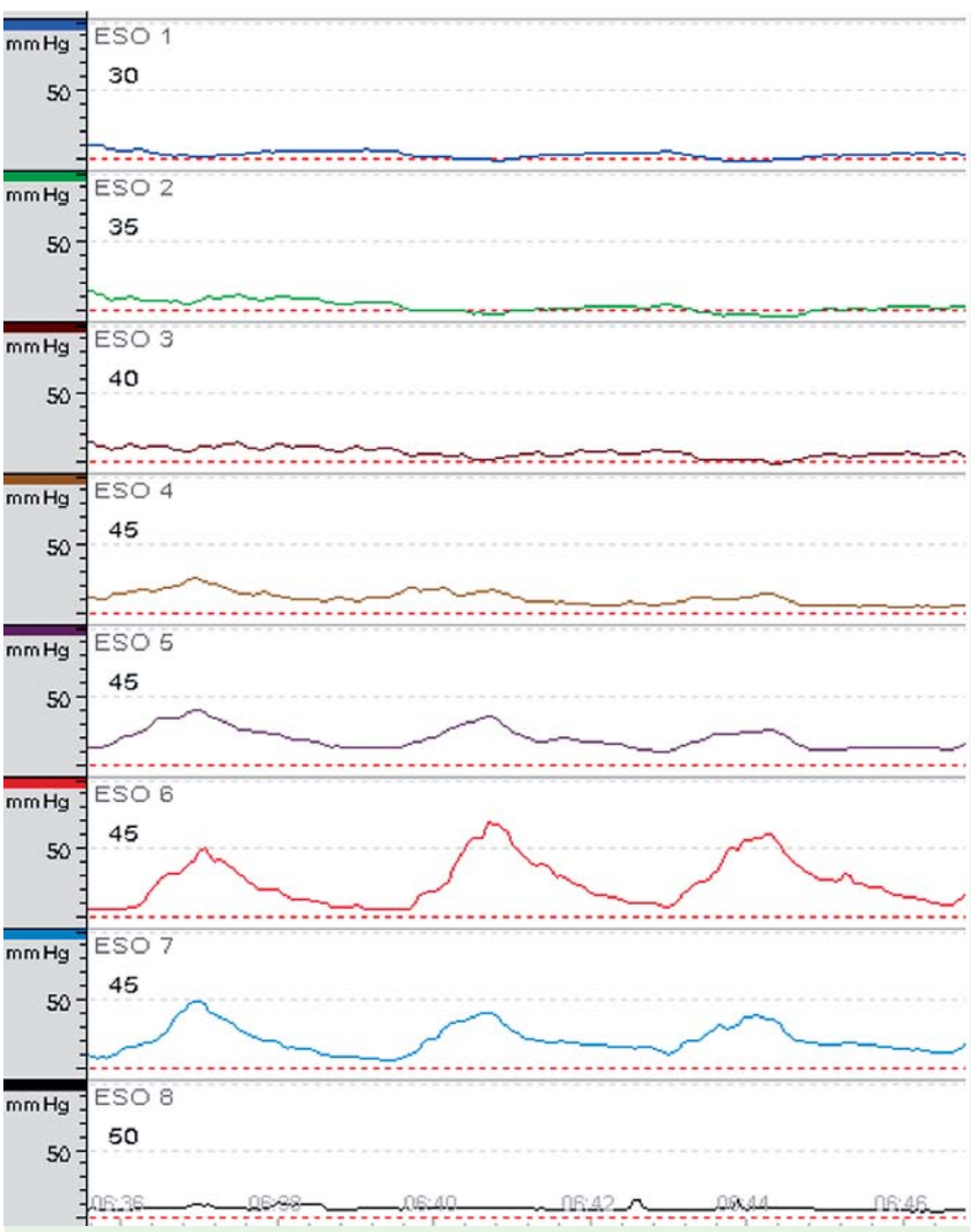

Fig. 4 The myotomy procedure reduced the pressure of the simultaneous contractions from 119.0 to $28.0 \mathrm{mmHg}$. 

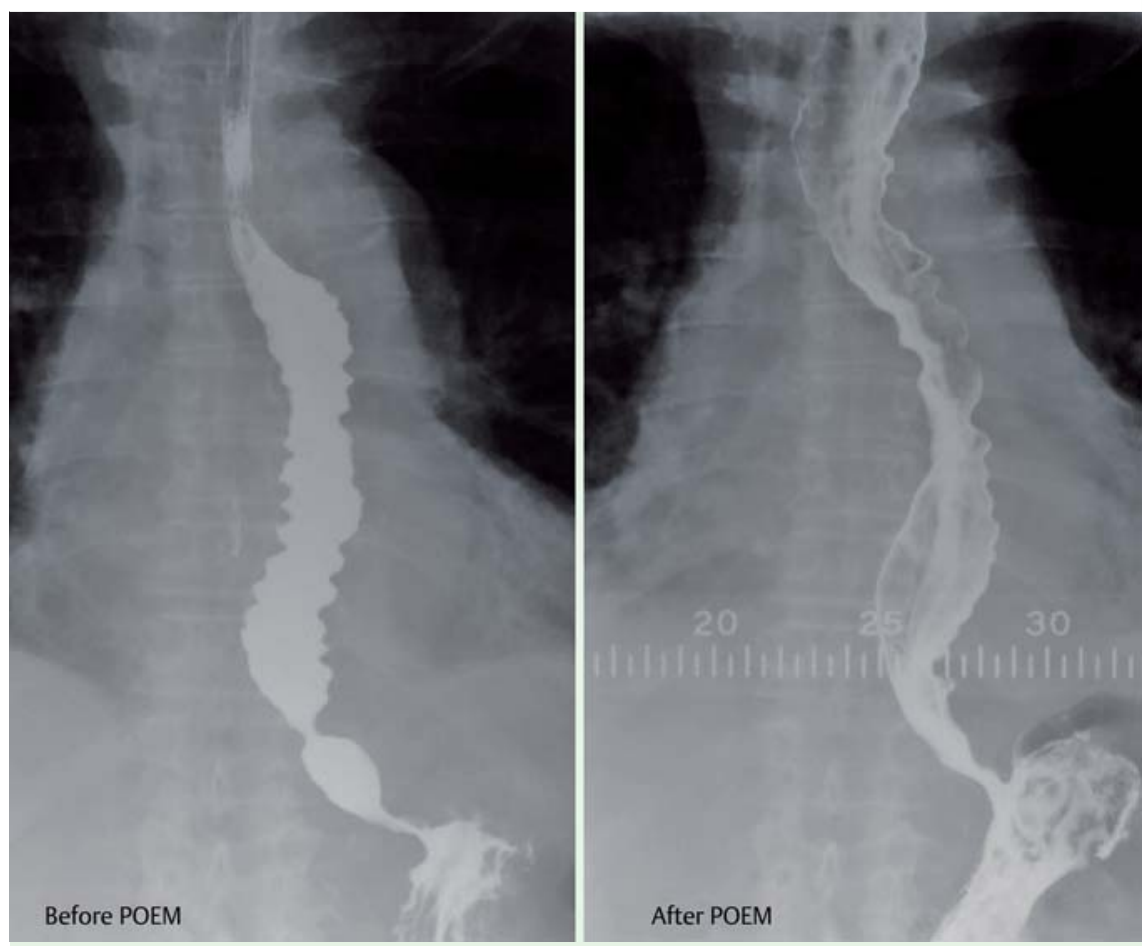

Endoscopy_UCTN_Code_TTT_1AO_2AD

Competing interests: None

\section{H. Minami ${ }^{1}$, H. Isomoto ${ }^{1}$, N. Yama- guchi ${ }^{1}$, K. Ohnita ${ }^{1}$, F. Takeshima ${ }^{1}$, H. Inoue ${ }^{2}$, K. Nakao'}

${ }^{1}$ Department of Gastroenterology and Hepatology, Nagasaki University Hospital, Nagasaki, Japan

${ }^{2}$ Digestive Disease Center, Showa University Northern Yokohama Hospital, Yokohama, Japan

\section{References}

1 Storr M, Allescher HD, Rosch T et al. Treatment of symptomatic diffuse esophageal spasm by endoscopic injection of botulinum toxin: a prospective study with long term follow-up. Gastrointest Endosc 2001; 54: $18 \mathrm{~A}$

2 Salvador R, Constantini M, Rizzetto $C$ et al. Diffuse esophageal spasm: the surgical approach. Dis Esophagus 2011; 4: 311-318 doi: $10.1111 / \mathrm{j} .1442-2050.2010 .01172 . x$

Fig. 5 Preoperative esophagogram showed severe and frequent simultaneous contractions, and barium barely passed the contractions. After the peroral endoscopic myotomy (POEM) procedure, barium passage was significantly improved with a complete release of muscle contraction on the anterior wall.

Table 1 Peroral endoscopic myotomy (POEM) in two patients for diffuse esophageal spasm. Maximum pressure and dysphagia scores were markedly improved.

\begin{tabular}{|c|c|c|}
\hline & Patient 1 & Patient 2 \\
\hline Age, years & 48 & 79 \\
\hline Operation time, minutes & 85.0 & 75.0 \\
\hline Myotomy length, $\mathrm{cm}$ & 15.0 & 10.0 \\
\hline \multicolumn{3}{|l|}{ Maximum pressure, $\mathrm{mmHg}$} \\
\hline Before POEM & 119.0 & 256.0 \\
\hline After POEM & 28.0 & 65.3 \\
\hline \multicolumn{3}{|l|}{ Dysphagia score, Ekcardt } \\
\hline Before POEM & 5 & 6 \\
\hline After POEM & 0 & 1 \\
\hline \multicolumn{3}{|l|}{ Dysphagia score, Vaezi } \\
\hline Before POEM & 6 & 7 \\
\hline After POEM & 0 & 0 \\
\hline Complications & None & None \\
\hline
\end{tabular}

3 Inoue H, Minami H, Kobayashi Y. Peroral endoscopic myotomy (POEM) for esophageal achalasia. Endoscopy 2010; 42: 265-271

4 Richter JE, Castell DO. Diffuse esophageal spasm: a reappraisal. Ann Intern Med 1984; 100: $242-245$

\section{Bibliography}

DOI http://dx.doi.org/

10.1055/s-0032-1309922

Endoscopy 2014; 46: E79-E81

(c) Georg Thieme Verlag KG

Stuttgart · New York

ISSN 0013-726X

\section{Corresponding author}

\section{H. Isomoto, MD, PhD}

Department of Gastroenterology and Hepatology Nagasaki University Hospital

1-7-1, Sakamoto

Nagasaki, 852-8501

Japan

Fax: +81-45-9497263

hajimei2002@yahoo.co.jp 